\title{
PRESENTACIÓN DE BACTERIAS RESISTENTES EN HEMOCULTIVOS EN PACIENTES CON PATOLOGÍA MÉDICA EN UN HOSPITAL DE TERCER NIVEL
}

Recibido: julio del 2018

Aceptado: marzo del 2019

Santiago Sánchez-Pardo ${ }^{1}$, Andrés Felipe Ochoa-Díaz ${ }^{2}$, Reynaldo Rodríguez ${ }^{3}$, Elsa Marina Rojas ${ }^{4}$

\section{Resumen}

Introducción. Las bacteriemias afectan a pacientes dentro del ámbito hospitalario y se reportan tasas de mortalidad del $35 \%$. Los microorganismos productores de beta-lactamasas de espectro extendido son cada vez más resistentes a los antibióticos. Materiales y métodos . Estudio observacional descriptivo de corte transversal en pacientes mayores de 14 años con hemocultivos positivos en el Hospital Universitario de Santander, entre 2014 y 2016. Resultados. Se analizaron 148 historia clínicas, la edad promedio fue de 55,5 años, el 51,4\% eran mujeres. La proporción de gérmenes resistentes fue del $23,6 \%$, la producción de beta-lactamasas de espectro extendido en E. coli y K. pneumoniae fue del $40 \%$. El $100 \%$ de los pacientes con gérmenes resistentes tenía un índice de PITT severo. El S. aureus mostró un $50 \%$ de resistencia a la oxacilina. Conclusión. El 23,6\% de los pacientes tenía una infección por gérmenes resistentes, los más comunes fueron E. coli y K. pneumoniae, la mortalidad general fue del $30 \%$.

Palabras clave: bacteriemia, enterobacteriaceae, farmacorresistencia bacteriana, infección hospitalaria.

${ }^{1}$ Especialización en Medicina Interna, Universidad industrial de Santander, Hospital Universitario de Santander.

2 Estudiante de VI año de Medicina, Universidad Industrial de Santander.

${ }^{3}$ Médico Magíster en Epidemiología, Universidad Industrial de Santander.

${ }^{4}$ Especialista en Medicina Interna e Infectología, Universidad Industrial de Santander. 


\section{PRESENTATION OF RESISTANT BACTERIA IN BLOOD CULTURES IN PATIENTS WITH MEDICAL PATHOLOGIES IN A THIRD LEVEL HOSPITAL}

Santiago Sánchez-Pardo ${ }^{1}$, Andrés Felipe Ochoa-Díaz ${ }^{2}$, Reynaldo Rodríguez ${ }^{3}$, Elsa Marina Rojas ${ }^{4}$

\section{Abstract}

Introduction: Bacteremia affects patients in the hospital, with mortality rates reported in $35 \%$. Microorganisms that produce betalactamases of extended spectrum are each time more resistant to antibiotics. Method: Observational and descriptive study in patients older tan 14 years old with positive blood cultures in Hospital Universitario de Santander, between 2014 and 2016. Results: 148 clinic records were analyzed, the mean age was 55.5 years. $51.4 \%$ were women. The proportion of resistant germs was of $23.6 \%$, the production of beta-lactamases of extended spectrum in E. coli and K. pneumoniae was $40 \% .100 \%$ of patients with resistant germs had a rate of severe PITT. S. aureus showed a 50\% of resistance to oxacillin. Conclusion: $23.6 \%$ of patients had an infection for resistant germs, the most common were E. coli and K. pneumoniae. General mortality was of $30 \%$. 


\section{APRESENTAÇ̃̃O DE BACTÉRIAS RESISTENTES EM HEMOCULTURAS EM PACIENTES COM PATOLOGIA MÉDICA EM UM HOSPITAL TERCIÁRIO}

Santiago Sánchez-Pardo ${ }^{1}$, Andrés Felipe Ochoa-Díaz ${ }^{2}$, Reynaldo Rodríguez ${ }^{3}$, Elsa Marina Rojas ${ }^{4}$

\section{Resumo}

Introdução: as bactérias afetam pacientes do contexto hospitalar e relatam taxas de mortalidade de $35 \%$. Os micro-organismos produtores de beta-lactamasas de espectro estendido são cada vez mais resistentes aos antibióticos. Materiais e métodos: estudo observacional descritivo de corte transversal com pacientes maiores de 14 anos com hemoculturas positivas, no Hospital Universitario de Santander, Colômbia, entre 2014 e 2016. Resultados: foram analisados 148 prontuários; a idade média foi de 55,5 anos; $51,4 \%$ eram mulheres. A proporção de germens resistentes foi de $23,6 \%$; a produção de beta-lactamasas de espectro estendido em E. coli e K. pneumoniae foi de $40 \% .100 \%$ dos pacientes com germens resistentes tinham um índice de PITT severo. O S. aureus mostrou $50 \%$ de resistência à oxacilina. Conclusão: $23,6 \%$ dos pacientes tinham uma infecção por germens resistentes, os mais comuns foram E. coli e K. pneumoniae; a mortalidade geral foi de $30 \%$. 


\section{Introducción}

Actualmente es evidente la necesidad del desarrollo de nuevos antimicrobianos, tal como lo manifiesta la Organización Mundial de la Salud (OMS) en el informe de desarrollo de nuevos antibióticos "Antibacterial agents in clinical development - An analysis of the antibacterial clinical development pipeline, including tuberculosis", donde se destacan 12 agentes infecciosos prioritarios para la investigación y además se resalta la poca disponibilidad de nuevos fármacos, ya que de 51 únicamente ocho son considerados como innovadores (1).

Dentro del gran espectro de las infecciones, las del torrente sanguíneo son un problema frecuente que a menudo afecta a pacientes que se encuentran dentro del medio hospitalario. Se estima que cada año en Estados Unidos se detectan 250000 casos con una mortalidad cercana al $35 \%$, con costos que superan los 35000 dólares americanos por paciente $(2,3)$.

Los organismos productores de beta-lactamasas de espectro extendido (BLEE), algunos de ellos causantes de infecciones frecuentes en la práctica clínica cotidiana como neumonía e infección de vías urinarias, son cada vez más resistentes a los antibióticos existentes y requieren con urgencia nuevos tratamientos, pues han sido reportados como agentes causales de brotes de infecciones multidrogorresistentes (MDR) y altas tasas de fracaso al tra- microorganismos como Escherichia coli y Klebsiella pneumoniae, estos son los productores de BLEE más comunes a nivel mundial y su número de aislamientos en hemocultivos ha venido creciendo, principalmente en pacientes con neoplasias hematológicas y neutropenia febril $(8,9)$. Llama la atención el hecho de que estos dos últimos patógenos que inicialmente eran descritos como patógenos nosocomiales, hoy en día son la causa del incremento en la incidencia de bacteriemia adquirida en la comunidad, situación que se acompaña de un incremento en los aislamientos de $S$. aureus resistente a la meticilina adquirido en la comunidad $(10,11)$, al tener en cuenta que este último microorganismo se asocia con peores resultados clínicos en distintos grupos de pacientes $(12,13)$.

Se conoce que América Latina tiene tasas de BLEE que superan el $20 \%$ de los casos, producidas principalmente por Enterobacteriaceae, S. aureus resistente a la meticilina (SARM) y Acinetobacter spp. MDR (14), sumándose de forma alarmante a este grupo K. pneumoniae productora de carbapenemasas (14-17). Es por lo anterior descrito que el objetivo del presente estudio es realizar una caracterización clínica y microbiológica de las infecciones del torrente sanguíneo por gérmenes resistentes en pacientes hospitalizados por patología médica del Hospital Universitario de Santander, aportando información que contraste con la ya publicada en otros escenarios y que permita orientar decisiones terapéuticas y diagnósticas. 


\section{Materiales y método}

\section{Diseño del estudio}

Se realizó un estudio observacional descriptivo, retrospectivo, tipo corte transversal, que incluyó únicamente los pacientes con hemocultivos positivos provenientes del servicio de medicina interna de un hospital de tercer nivel de complejidad de la ciudad de Bucaramanga, Colombia, entre los años 2014 y 2016. Se incluyeron hombres y mujeres mayores de 14 años que cumplieran los criterios del Centers for Disease Control and Prevention (CDC) para infección del torrente sanguíneo.

Se excluyeron los servicios del hospital diferentes a medicina interna (ginecología, cirugía y ortopedia), obteniéndose un total de 926 registros de hemocultivos positivos para 2014, 1.029 para 2015 y 1.050 para 2016. Del compilado anterior, se revisaron un total de 450 historias clínicas, obteniéndose al final un total de 148 pacientes finales para el análisis posterior a la exclusión de los pacientes de la unidad de cuidado intensivo, unidad de hematología con enfermedad hematológica activa o con necesidad de quimioterapia y aquellos con dos o más ingresos hospitalarios durante el periodo de observación.

El registro de los aislamientos y los patrones de resistencia se llevó a cabo mediante el software Whonet que utiliza el Hospital Universitario de Santander. Las pruebas de sensibilidad a los antibióticos se realizaron mediante métodos estandarizados de difusión y sistemas automatizados (Phoenix 1000 ${ }^{\mathrm{TM}}$ de Bec-
tonDickinson $^{\circledast}$ ) en concordancia con los criterios del Clinical and Laboratory Standards Institute (CLSI), los cuales se verificaron usando los puntos de corte de la versión 2017 según referencia del laboratorio clínico de la institución.

\section{Análisis estadístico}

El análisis estadístico se realizó mediante un análisis descriptivo de cada una de las variables a estudio. La información se presenta mediante estadísticas descriptivas (medidas de dispersión y de tendencia central) y se muestra en texto, tablas o gráficas, empleando software como Excel y Stata 2.0.

Se midieron importantes variables clínicas y de laboratorio para el desenlace de interés, para las variables cuantitativas de laboratorio se realizó una prueba de ShapiroWilk, con el fin de determinar su distribución normal, con expresión en medias y rangos intercuartílicos. Las variables cualitativas se expresaron en porcentajes, se realizaron comparaciones de proporciones entre el grupo de pacientes con aislamientos resistentes y aquellos con aislamientos no resistentes.

\section{Consideraciones éticas}

Este fue un estudio retrospectivo que utilizó datos extraídos de las historias clínicas de pacientes, por lo tanto, se consideran "sin riesgo" de acuerdo con la clasificación establecida en la Resolución 8430 de 1993 del Ministerio de Salud, y se condujo de acuerdo con los principios declarados en la XVIII Asamblea Médica Mundial (Helsinki, 1964). El protocolo fue aprobado por el 
comité de ética hospitalaria del centro asistencial y el comité de ética en investigación de la Universidad Industrial de Santander.

\section{Resultados}

Se analizaron 450 historias clínicas, de las cuales se incluyeron 148 (32,8\%) para el análisis. De estas últimas, el $65,5 \%(n=97)$ presentaron infecciones por gérmenes potencialmente resistentes que a su vez ocuparon los primeros lugares dentro de los más frecuentes.

El 23,6\% $(n=35)$ de todos los pacientes presentaban infecciones con bacterias con perfiles amplios de resistencia, incluidos E. coli y K. pneumoniae productores de BLEE en un $40 \%$, SARM $25,7 \%$, A. baumanii MDR 22,8\% y $P$. aureginosa MDR 11,4\%. Se definieron las categorías de MDR de acuerdo a la clasificación propuesta por Magiorakos, et al. (18), donde un microorganismo se caracteriza por ser resistente a por lo menos un antibiótico en más de tres categorías de medicamentos, los cuales incluyen amino-glucósidos, carbapenémicos, cefamicinas, fluoroquinolonas, inhibidores de la síntesis del folato, etcétera. Para el caso puntual de $S$. aureus, este se considera como MDR por ser meticilino-resistente o tener resistencia a más de un antimicrobiano en más de tres categorías de medicamentos, al igual que las enterobacterias (18).
El promedio de edad de aquellos pacientes que presentaban algún grupo de bacterias resistentes fue de 55,5 años (de 18,2 años), y el de los que no presentaban bacterias resistentes fue de 57,6 años $(\mathrm{p}=0,5)$. De los pacientes con gérmenes resistentes, el 51,4\% eran mujeres. Uno de los antecedentes más importantes fue el de infección por virus de la inmunodeficiencia humana (VIH) con un total de 38 casos, de los cuales la mayoría fueron diagnósticos nuevos sin tratamiento antirretroviral, el $27 \%$ tenían infecciones por gérmenes resistentes y cercal del $92 \%$ poseían recuentos de linfocitos $\mathrm{T}$ CD 4 < a 200 células $/ \mathrm{mm}^{3}$.

De los pacientes que presentaron aislamientos de gérmenes resistentes, el $37,8 \%$ habían estado hospitalizados previamente con un promedio de 11,7 días. La mortalidad general fue del $30 \%$, sin embargo, no hubo diferencias estadísticamente significativas entre los grupos de resistentes y no resistentes.

El 85,8\% de los pacientes recibieron manejo antibiótico empírico, de los cuales el 59,7\% recibieron monoterapia, 21,6\% terapia dual y el $4,1 \%$ tres o más antibióticos. Los antibióticos empíricos más frecuentemente utilizados fueron piperacilina tazobactam en un $47,4 \%$; vancomicina $28,8 \%$; meropenem $17,5 \%$; ceftriaxona $4 \%$; ampicilina sulbactam $2 \%$; linezolid $2 \%$; y oxacilina $1 \%$. Las variables cualitativas y cuantitativas se presentan en la tabla 1. 
Tabla 1. Comparación de variables cualitativas y cuantitativas de los pacientes con infecciones por microorganismos resistentes y no resistentes

\begin{tabular}{|c|c|c|}
\hline Variables cuantitativas & $\begin{array}{c}\text { Pacientes con } \\
\text { infecciones por } \\
\text { bacterias resistentes } \\
(n=35)\end{array}$ & $\begin{array}{c}\text { Pacientes con } \\
\text { infecciones por } \\
\text { bacterias no } \\
\text { resistentes }(n=62)\end{array}$ \\
\hline Edad (años) & 55,5 & 57,6 \\
\hline Hospitalización previa (\%) & 37,8 & 62,1 \\
\hline Tiempo de hospitalización previa (días) & 11,7 & 13,7 \\
\hline Tiempo de estancia hospitalaria (días) & 48,9 & 40 \\
\hline $\begin{array}{l}\text { Tiempo estancia en el servicio de } \\
\text { urgencias (días) }\end{array}$ & 5,1 & 5,1 \\
\hline Hemocultivos positivos & 2 & 2 \\
\hline $\begin{array}{l}\text { Creatinina al momento de los } \\
\text { hemocultivos (mg/dL) }\end{array}$ & 2,8 & 2,4 \\
\hline $\begin{array}{l}\text { Variables cualitativas / } \\
\text { categóricas }\end{array}$ & $\begin{array}{c}\text { Pacientes con } \\
\text { infecciones por } \\
\text { bacterias resistentes }\end{array}$ & $\begin{array}{l}\text { Pacientes con } \\
\text { infecciones por } \\
\text { bacterias no } \\
\text { resistentes } \\
\end{array}$ \\
\hline $\begin{array}{l}\text { Leucocitos al momento de la toma de } \\
\text { los hemocultivos mayores a } 12000 \mathrm{cel} / \\
\mathrm{mm}^{3}(\%)\end{array}$ & 37,8 & 62,1 \\
\hline Anemia (\%) & 38,5 & 61,4 \\
\hline Fiebre (\%) & 28,5 & 71,4 \\
\hline $\begin{array}{l}\text { Índice de mortalidad de PITT } \\
\text { moderado (\%) }\end{array}$ & 33,3 & 66,6 \\
\hline Índice de mortalidad de PITT severo \%) & 100 & 0 \\
\hline Sitio primario piel y tejidos blandos (\%) & 28 & 72 \\
\hline Sitio primario sistema urinario (\%) & 47 & 53 \\
\hline Sitio primario sistema vascular (\%) & 45 & 55 \\
\hline $\begin{array}{l}\text { Infección clasificada mayor a } 48 \text { horas } \\
\text { del ingreso (\%) }\end{array}$ & 33,7 & 66,3 \\
\hline Muerte (\%) & 39 & 61 \\
\hline $\begin{array}{l}\text { Cultivos con aislamientos poli } \\
\text { icrobianos (\%) }\end{array}$ & 31,2 & 68,7 \\
\hline
\end{tabular}

Fuente: elaboración propia. 
La distribución de las bacterias resis- clínicas entre las enterobacterias y el retentes se presenta en la gráfica 1 y sus presentante de los gérmenes gram posiperfiles de resistencia en las tablas 2 y 3 , tivos S. aureus. donde se tienen en cuenta las diferencias

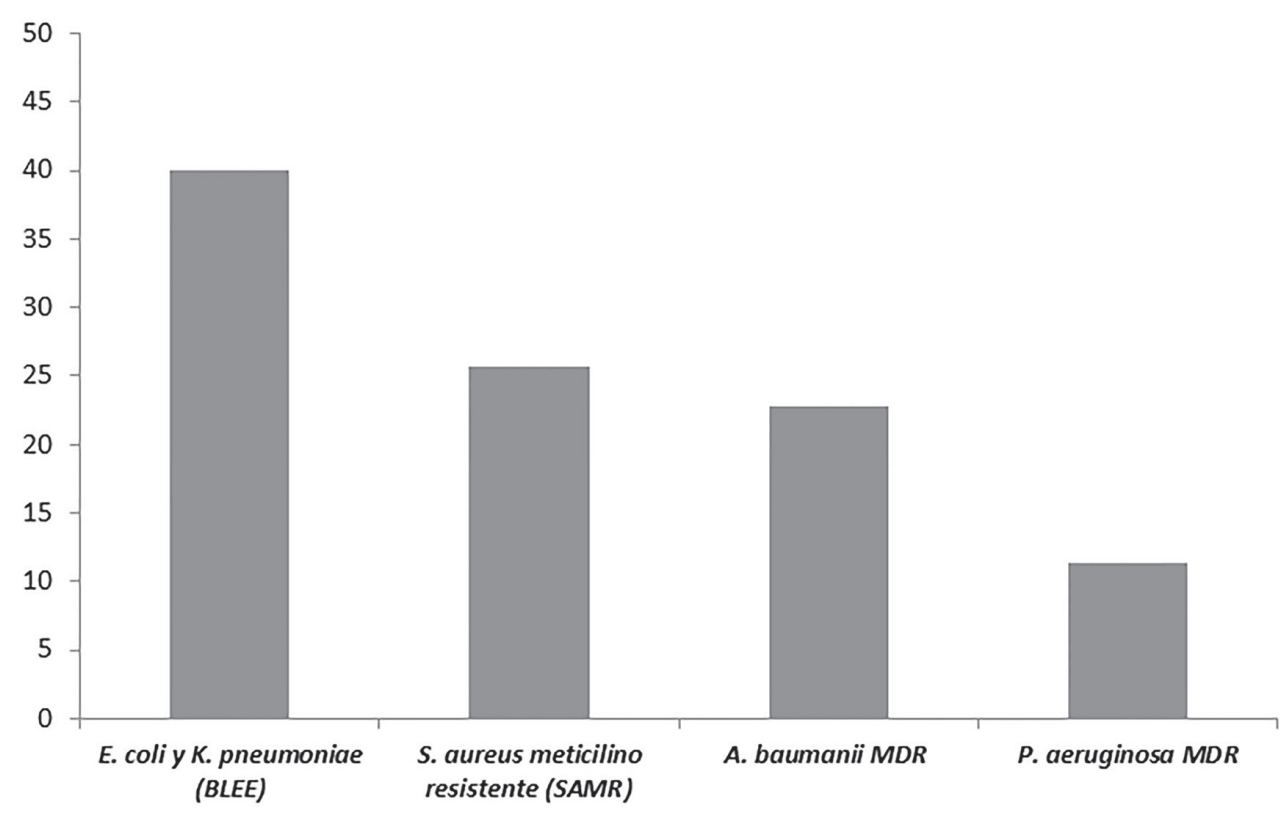

BLEE: Beta-Lactamasas de Espectro Extendido.

Gráfica 1. Distribución de los microorganismos con perfiles amplios de resistencia Fuente: elaboración propia.

Tabla 2. Perfiles de resistencia a los antibióticos de interés clínico

\begin{tabular}{l|c|c|c|c}
\hline $\begin{array}{c}\text { Antibiótico \% de } \\
\text { resistencia }\end{array}$ & $\begin{array}{c}\text { K. pneumoniae } \\
\text { (\%) }\end{array}$ & E. coli (\%) & $\begin{array}{c}\text { A. baumanii } \\
\text { (\%) }\end{array}$ & $\begin{array}{c}\text { P. aeruginosa } \\
\text { (\%) }\end{array}$ \\
\hline Ceftazidime & 29,6 & 25 & 38,8 & 26,6 \\
Cefepime & 19,2 & 9 & 53 & 28,5 \\
Cefoxitin & 25,9 & 18,1 & $\mathrm{NA}$ & $\mathrm{NA}$ \\
Aztreonam & 48,1 & 16,6 & 66,6 & 20 \\
Ciprofloxacina & 55,5 & 22,2 & 58,8 & 50 \\
Gentamicina & 50 & 0 & 50 & 22 \\
Meropenem & 16,6 & 12,5 & 46,1 & 30 \\
Piperacilina tazobactam & 33,3 & 14,2 & 50 & 20 \\
Trimetropim sulfametoxazol & 58,8 & 14,2 & 46,6 & $\mathrm{NA}$ \\
Ampicilina sulbactam & 0 & 0 & 0 & 0 \\
\hline
\end{tabular}

NA: No aplica por resistencia natural.

Fuente: elaboración propia. 
Tabla 3. Resistencia a los antibióticos de interés clínico de S. aureus

\begin{tabular}{lc|}
\hline \multicolumn{2}{c}{ Staphylococcus aureus } \\
\hline Antibiótico & Resistencia (\%) \\
\hline Ciprofloxacina & 20 \\
Gentamicina & 15,3 \\
Trimetropim sulfametoxazol & 0 \\
Clindamicina & 11,7 \\
Oxacilina & 50 \\
Vancomicina & 0 \\
Rifampicina & 0 \\
Linezolid & 0 \\
Daptomicina & 0 \\
\hline
\end{tabular}

Fuente: elaboración propia.

Al tener en cuenta que los pacientes fue- llazgos con respecto al aislamiento de ron atendidos en un servicio de medici- bacterias resistentes, teniendo en cuenta na interna y la diversidad de sus patolo- que un paciente podía tener más de un gías, se presentan en la tabla 4 los sitios sitio anatómico como fuente de infecanatómicos fuentes de la infección más ción o aislamiento del mismo microorfrecuentemente documentados y los ha- ganismo en más de un sitio.

Tabla 4. Fuente primaria de la infección y aislamientos de bacterias resistentes

\begin{tabular}{|c|c|c|c|c|c|}
\hline \multicolumn{6}{|l|}{$N=97$} \\
\hline $\begin{array}{l}\text { Fuente de la } \\
\text { infección }\end{array}$ & $\begin{array}{c}\text { E. coli y K. } \\
\text { pneumoniae } \\
\text { (BLEE) }\end{array}$ & $\begin{array}{l}\text { A. baumanii } \\
\text { (MDR) }\end{array}$ & $\begin{array}{c}\text { P. aureginosa } \\
\text { (MDR) }\end{array}$ & $\begin{array}{l}\text { S. aureus } \\
\text { Meticilino R } \\
\text { (SARM) }\end{array}$ & Total \\
\hline Vascular & 2 & 4 & 2 & 6 & 14 \\
\hline Urinario & 7 & 4 & 3 & 4 & 18 \\
\hline Respiratorio & 3 & 2 & 1 & 1 & 7 \\
\hline $\begin{array}{l}\text { Piel y tejidos } \\
\text { blandos }\end{array}$ & 5 & 3 & 0 & 0 & 8 \\
\hline Abdomen & 6 & 0 & 1 & 0 & 7 \\
\hline
\end{tabular}




\section{Discusión}

El presente fue un estudio realizado por primera vez en el nororiente colombiano en pacientes con patología médica, diferente a otras series donde se han incluido pacientes en contextos diferentes al expuesto, tales como necesidad de quimioterapia, trasplante de médula ósea, en servicios de urgencias o la unidad de cuidados intensivos (UCI), tanto en Colombia como en otras latitudes (9, 19-23); sin embargo, se demuestra que la resistencia bacteriana se ha extendido de estos contextos a los demás escenarios de los hospitales e incluso se ha diseminado al contexto ambulatorio y comunitario $(10,11)$.

La mortalidad general se acerca a otros estudios que han documentado infecciones por microorganismos resistentes de índole local, como el estudio de Navarro-Oliveros, et al., realizado en $\mathrm{Me}$ dellín, Colombia, donde la mortalidad relacionada con la bacteriemia por enterobacterias productoras de carbapenemasas fue del $40 \%$ (9), cuestión que es preocupante pues representa una cifra bastante alta y dado que los mecanismos de resistencia son difíciles de diagnosticar por los métodos estandarizados usuales se debe procurar la utilización de técnicas de laboratorio diferentes como los métodos de biología molecular o reacción en cadena de la polimerasa (RCP).

Llama la atención el cambio actual de los aislamientos microbiológicos a diferencia de lo que ocurría en la década de los 90. En esta última, gérmenes como
S. aureus ocupaban el primer lugar y actualmente han cedido su puesto a las enterobacterias, situación que se evidencia en la UCI según un estudio de Díaz, et al., en Perú (25), donde estas incluso en muestras diferentes a hemocultivos ocupan los primeros lugares.

Se han descrito factores de riesgo para la adquisición exógena o endógena de bacterias resistentes, los de la primera se asocian usualmente a la transmisión por contacto con las manos contaminadas de los trabajadores de la salud y la contaminación de superficies ambientales o de dispositivos médicos por procesos inadecuados de limpieza y desinfección (la mortalidad aumenta principalmente con la emergencia de microorganismos multirresistentes y al tratamiento antibiótico inadecuado); los factores de la adquisición endógena, tales como los propios del paciente (edad, estado nutricional y enfermedades concomitantes) y de la infección (aparición temprana o tardía, tipo, localización y compromiso) influyen a la vez de forma importante en este desenlace. A pesar de que no era un objetivo del estudio, vale la pena realizar estudios con más variables clínicas a las descritas, con el fin de tratar de determinar más claramente estas asociaciones en un ámbito clínico diferente $(2,6,8)$.

Por otro lado, es llamativa la presencia de resistencia a carbapenémicos del $17 \%$ de K. pneumoniae, no obstante, no se pudo estimar asociación alguna de esta con el desenlace mortalidad o entre esta con el haber recibido uno u otro antibiótico, dado que no consistía en un objetivo del estudio; en cambio, permite evidenciar que una vez las entero- 
bacterias desarrollan BLEE, fácilmente pueden desarrollar resistencia a los carbapenémicos, limitando así las opciones terapéuticas $(16,20,24)$.

Es importante resaltar que uno de los antecedentes más frecuentes fue la infección por VIH y de estos pacientes la mayoría fueron diagnósticos nuevos y, por ende, sin tratamiento antirretroviral, lo que deja ver que el departamento de Santander continúa siendo uno de los primeros en la detección y notificación de esta entidad (26) y que las infecciones bacterianas están emergiendo como causa de mortalidad sumado a los oportunismos clásicamente descritos (27).

En este estudio se encontró que la utilización de antibióticos empíricos es superior al $80 \%$, lo que demuestra lo difícil del diagnóstico y la necesidad de iniciar antibioticoterapia oportunamente; adicionalmente, el hecho de que la mayoría de los antibióticos usados eran de amplio espectro puede acarrear consecuencias en la resistencia bacteriana a futuro. Las diferencias de porcentaje de resistencia según diversos estudios son escasas, a pesar de que estos han sido realizados en escenarios distintos a paciente con bacteriemia (28). Por otro lado, no se encontraron diferencias estadísticamente significativas entre las variables cualitativas y cuantitativas entre los grupos, no obstante, esto pudo deberse el tamaño de muestra y además no era uno de los objetivos del estudio.

A pesar de las limitaciones ya mencionadas, este estudio permitió identificar que los gérmenes MDR son una preocu- pación creciente, la cual deben llevar a un adecuado uso e implementación de los programas o estrategias con el fin de modificar ciertas condiciones y así reducir la mortalidad en los pacientes con infecciones asociadas a la atención en salud. Dichas estrategias incluyen el uso adecuado de antibióticos, lo que se logra a través de programas de vigilancia, control, protocolización e intervención en el uso de antibióticos (antibiotic stewardship) y el empleo de antibióticos que garanticen una respuesta terapéutica equivalente a la de las moléculas innovadoras $(29,30)$.

En concordancia con lo anterior y proponiendo escenarios ideales en los que se cuente con moléculas innovadoras de forma permanente y un adecuado uso racional de antimicrobianos, un estudio publicado por Rattanaumpawan, et al. (31) propone el uso de ertapenem como estrategia de ajuste terapéutico para pacientes estables con infecciones por enterobacterias productoras de BLEE y el uso de meropenem en aquellos críticamente enfermos, como una opción terapéutica mientras se cuenta con el concepto del especialista en enfermedades infecciosas y así prevenir la emergencia de resistencia sin afectar el desenlace clínico.

La mortalidad de estos pacientes continúa siendo elevada con un porcentaje cercano al $30 \%$ en el presente estudio, porcentaje que concuerda con la literatura disponible al tener en cuenta que la mayoría son estudios realizados en otros escenarios clínicos (2, 32-35). Es de esta manera que se ratifica que la presencia de bacteriemia le confiere 
peor desenlace a los pacientes hospitalizados con patologías médicas y que se asemeja a pacientes hospitalizados por patologías quirúrgicas, sobre quienes originalmente se documentó esta entidad, secundario a procesos sépticos de origen abdominal $(35,36)$.

\section{Conclusiones}

A pesar de ser un estudio retrospectivo, los hallazgos son de gran relevancia ya que dejan ver cómo la proporción de resistencia a los antibióticos viene en aumento, incluso en pacientes hospitalizados por patologías médicas fuera de escenarios como la UCI o las unidades hematológicas. El $23 \%$ de aquellos pacientes con bacteriemia contaban con gérmenes de alta resistencia, siendo $E$. coli y K. pneumoniae BLEE los responsables del $40 \%$ de las mismas. Es preocupante cómo las estrategias terapéuticas cada vez son menores, por lo que se deben implementar medidas de control y de uso racional de antimicrobianos o fortalecer las ya existentes en las instituciones prestadoras de salud.

\section{Agradecimientos}

Agradecemos a los pacientes por permitir ser objeto de este estudio y al Hospital Universitario de Santander.

\section{Conflicto de intereses}

Los autores declaran no tener ningún conflicto de interés para la realización del presente estudio.

\section{Fuentes de financiación}

Los autores declaran no tener ninguna fuente de financiación para la realización del presente estudio; este último se realizó con recursos propios. 


\section{Referencias bibliográficas}

1. World Health Organization. Antibacterial agents in clinical development an analysis of the antibacterial clinical development pipeline, including tuberculosis [Internet]. Geneva: World Health Organization; 2017 [citado 2017 nov. 25]. Disponible en: http://www.who.int/iris/ handle/10665/258965

2) Pallares CJ, Martínez E. Factores de riesgo asociados a mortalidad en infecciones relacionadas con la atención en salud en un hospital universitario de tercer nivel en Colombia. Biomédica. 2014;34(Suppl 1):148-55.

3. Hugonnet S, Sax H, Eggimann $P$, Chevrolet JC, Pittet D. Nosocomial bloodstream infection and clinical sepsis. Emerging Infectious Diseases. 2004;10(1):76-81.

4. Park YS, Bae II K, Kim J, Jeong SH, Hwang SS, Seo Y, et al. Risk factors and molecular epidemiology of community-onset extended-spectrum $\beta$-lactamase-producing Escherichia coli bacteremia. Yonsei Med J. 2014;55(2):467-75.

5. Pitout JD, Laupland KB. Extended-spectrum beta-lactamase-producing Enterobacteriaceae: an emerging public health concern. Lancet Infect Dis. 2008;8(3):159-66.

6. Tuon FF, Kruger M, Terreri M, Penteado-Filho SR, Gortz L. Klebsiella ESBL bacteremia-mortality and risk factors. Braz J Infect Dis. 2011;15(6):594-8.

7. Kaya O, Akcam FZ, Gonen I, Unal O, Ceylan T. Risk factors for bacteremia due to extended-spectrum beta-lactamase-producing Escherichia coli in a Turkish hospital. J Infect Dev Ctries. 2013;7(7):507-12.

8. Valderrama SL, González PF, Caro MA, Ardila N, Ariza B, Gil F, et al. Factores de riesgo para bacteriemia adquirida en el hospital por Pseudomonas aeruginosa resistente a carbapenémicos en un hospital colombiano. Biomédica. 2016;36(Suppl 1):69-77.

9. Kim SH, Kwon JC, Choi SM, Lee DG, Park SH, Choi JH, et al. Escherichia coli and Klebsiella pneumoniae bacteremia in patients with neutropenic fever: factors associated with extended-spectrum $\beta$-lactamase production and its impact on outcome. Ann Hematol. 2013;92(4):533-41.

10. Hanses F, Spaeth C, Ehrenstein BP, Linde HJ, Schölmerich J, Salzberger B. Risk factors associated with long-term prognosis of patients with Staphylococcus aureus bacteremia. Infection. 2010;38(6):465-70.

11. Barrero LI, Castillo JS, Leal AL, Sánchez R, Cortés JA, Álvarez CA, et al. Impacto económico de la resistencia a la meticilina en pacientes con bacteriemia por Staphylococcus aureus en hospitales de Bogotá. Biomédica. 2014;34(3):345-53.

12. Lasa JS, Fernández ML, Finn BC, Bruetman JE, Peroni J, Young P. Bacteriemia en pacientes internados con celulitis. Medicina. 2011;71(4):298-304.

13. Cosgrove SE, Sakoulas G, Perencevich EN, Schwaber MJ, Karchmer AW, Carmeli Y. Comparison of mortality associated with methicillin-resistant and methicillin-susceptible Staphylococcus aureus bacteremia: A meta-analysis. Clin Infect Dis. 2003;36(1):53-9.

14. Vega S, Dowzicky J M. Antimicrobial susceptibility among gram-positive and gram-negative organisms collected from the Latin American region between 2004 and 2015 as part of the Tigecycline Evaluation and Surveillance Trial. Ann Clin Microbiol Antimicrob. 2017;16(1):50.

15. Jones RN, Guzman M, Gales AC, Gallegos B, Castrol AL, Martino MD, et al. Susceptibility rates in Latin American nations: report from a regional resistance surveillance program (2011). Braz J Infect Dis. 2013;17(6):672-81. 
16. Guzmán M, Labarca JA, Villegas MV, Gotuzzo E. Latin America working group on bacterial resistance: Extended spectrum $\beta$-lactamase producers among nosocomial enterobacteriaceae in Latin America. Braz J Infect Dis. 2014;18(4):421-33.

17. Biedenbach DJ, Hoban DJ, Reiszner E, Lahiri SD, Alm RA, Sahm DF, et al. In vitro activity of ceftaroline against Staphylococcus aureus isolates collected in 2012 from Latin American countries as part of the AWARE surveillance program. Antimicrob Agents Chemother. 2015;59(12):7873-7.

18. Magiorakos AP, Srinivasan A, Carey RB, Carmeli Y, Falagas ME, Giske CG, et al. Multidrug-resistant, extensively drug-resistant and pandrug-resistant bacteria: an international expert proposal for interim standard definitions for acquired resistance. Clin Microbiol Infect. 2012;18(3):268-81.

19. Horan TC, Andrus M, Dudeck MA. $\mathrm{CDC} / \mathrm{NHSN}$ surveillance definition of health care-associated infection and criteria for specific types of infections in the acute care setting. Am J Infect Control. 2008;36(5):309-32.

20. Kang Cl, Chung DR, Ko KS, Peck KR, Song JH. Risk factors for infection and treatment outcome of extended-spectrum $\beta$-lactamase-producing Escherichia coli and Klebsiella pneumoniae bacteremia in patients with hematologic malignancy. Ann Hematol. 2012;91(1):115-21.

21. Ye QF, Zhao J, Wan QQ, Qiao BB, Zhou JD. Frequency and clinical outcomes of ESKAPE bacteremia in solid organ transplantation and the risk factors for mortality. Transpl Infect Dis. 2014;16(5):767-74.

22. Piñana JL, Montesinos $\mathrm{P}$, Martino $R$, Vázquez L, Rovira $M$, López $J$, et al. Incidence, risk factors, and outcome of bacteremia following autologous hematopoietic stem cell transplanta-
23. Freifeld AG, Bow EJ, Sepkowitz KA, Boeckh MJ, Ito JI, Mullen CA, et al. Clinical practice guideline for the use of antimicrobial agents in neutropenic patients with cancer: 2010 update by the Infectious Diseases Society of America. Clinical Infectious Diseases. 2011;52(4):e56-93.

24. Navarro AO, Uribe N, Sierra P, Jaimes F, González JM. Bacteriemia por enterobacterias resistentes a carbapenems. Un estudio transversal. Infectio. 2015;19(2):60-6.

25. Díaz J, Rojas J, Ibarra J, Tárraga D. Sensibilidad antimicrobiana de la microbiota ambiental de las unidades de cuidados intensivos de un hospital peruano. Revista Peruana de Medicina Experimental y Salud Pública. 2017;34(1):93-7.

26. Instituto Nacional de Salud. Comportamiento del VIH/SIDA en Colombia a semana epidemiológica 44, 2016, dirección de vigilancia y análisis del riesgo en salud pública [Internet]. Colombia: Ministerio de Salud; 2016 [citado 2017 dic. 13]. Disponible en: http://www.ins.gov.co/ noticias/reunin $\% 20$ nacional $\% 20 \mathrm{de} \% 20$ vigilancia $\% 20$ en $\% 20$ salud $\% 20$ publica/27. $\% 20$ vigilancia $\% 20$ de $\% 20 l a s \% 20$ its.pdf

27. O'Connor J, Vjecha JM, Phillips NA, Angus B, Cooper D, Grinsztejn B, et al. Effect of immediate initiation of antiretroviral therapy on risk of severe bacterial infections in HIV-positive people with CD4 cell counts of more than 500 cells per $\mu \mathrm{L}$ : secondary outcome results from a randomised controlled trial. Lancet HIV. 2017;4(3):e105-12.

28. Quijada P, Flores A, Labrador I, Araque M. Estudio clínico y microbiológico de la infección urinaria asociada a catéter en los servicios de medicina interna de un hospital universitario venezolano. Revista Peruana de Medicina Experimental y Salud Pública. 2017;34(1):52-61.

29. Kollef $\mathrm{MH}$. Inadequate antimicrobial treatment: an important determinant of 2014;93(2):299-307. 
outcome for hospitalized patients. Clin Infect Dis. 2000;31(Suppl 4):S131-8.

30. Dellit HT, Owens CR, McGowan JE, Gerding DN, Weinstein RA, Burke JP, et al. Infectious diseases Society of America and the Society for Healthcare Epidemiology of America guidelines for developing an institutional program to enhance antimicrobial stewardship. Clinical Infectious Diseases. 2007;44(2):159-77.

31. Rattanaumpawan $P$, Werarak $P$, Jitmuang A, Kiratisin P, Thamlikitkul V. Efficacy and safety of de-escalation therapy to ertapenem for treatment of infections caused by extended-spectrum- $\beta$-lactamase-producing Enterobacteriaceae: an open-label randomized controlled trial. BMC Infect Dis. 2017;17(1):183.

32. Cortes JA, Leal AL, Montañez AM, Buitrago G, Castillo JS, Guzman L. Frequency of microorganisms isolated in patients with bacteremia in intensive care units in Colombia and their resistance profiles. Braz J Infect Dis. 2013;17(3):346-52.

33. González AL, Leal AL, Cortés JA, Sánchez R, Barrero LI, Castillo JS, et al.
Efecto del tratamiento antibiótico inicial adecuado sobre la mortalidad en pacientes en estado crítico con bacteriemia por Pseudomonas aeruginosa. Biomédica. 2014;34(Suppl 1):58-66.

34. Cortés JA, Garzón DC, Navarrete JA, Contreras KM. Impact of inappropriate antimicrobial therapy on patients with bacteremia in intensive care units and resistance patterns in Latin America. Rev Argent Microbiol. 2010;42(3):230-4 .

35. Castillo JS, Leal AL, Cortes JA, Álvarez $C A$, Sánchez $R$, Buitrago $G$, et al. Mortality among critically ill patients with methicillin-resistant Staphylococcus aureus bacteremia: a multicenter cohort study in Colombia. Rev Panam Salud Pública. 2012;32(5):343-50.

36. Abraham K, Dolman HS, Zimmerman LH, Faris J, Edelman DA, Baylor A. Impact of inappropriate initial antibiotics in critically ill surgical patients with bacteremia. The American Journal of Surgery. 2016;211(3):593-8. 